\title{
Lesson of the month 1: Seeing snowflakes
}

\author{
Authors: Anand Patel, ${ }^{A}$ Arif Aslam, ${ }^{B}$ Gavin Orr $^{C}$ and William Perkins ${ }^{D}$
}

Melanoma-associated retinopathy is a rare paraneoplastic complication of metastatic cutaneous malignant melanoma. It may present years after the original diagnosis of melanoma. We describe a patient with this condition who presented with persistent visual symptoms.

KEYWORDS: Photopsias, electroretinography, melanomaassociated retinopathy

\section{Introduction}

Paraneoplastic syndromes are caused by the immunological effects of a neoplasm located remotely from the affected organ. Melanoma-associated retinopathy (MAR) is a paraneoplastic retinal disorder occurring in patients with cutaneous melanoma. Diagnosing MAR is challenging because of its rarity and subtle ophthalmoscopic findings. The absence of a history of melanoma cannot rule out MAR because it may precede or follow the diagnosis and is not always associated with metastatic disease.

\section{Case report}

A 59 year-old male with Fitzpatrick skin phototype I presented to our department with two changing melanocytic naevi located on the left and right forearm at almost identical sites. Excision was arranged and histological examination confirmed a 1-mm thick melanoma on the left forearm and a melanoma in situ on the right forearm. Appropriate excision margins were instigated. Eleven months later the patient reported visual disturbances described as 'snowflakes' obscuring his vision. He described interference of peripheral vision with visual shimmering and a change in colour perception. He had no other past medical history or any systemic symptoms. He was referred to ophthalmology where his acuity tested normal. On the basis of his visual

Authors: A consultant dermatologist and dermatological surgeon, Department of Dermatology, Nottingham NHS Treatment Centre, Nottingham, UK; ${ }^{B}$ post-CCT fellow in Mohs micrographic surgery, Department of Dermatology, Nottingham NHS Treatment Centre, Nottingham, UK; ${ }^{C}$ consultant ophthalmologist, Department of Ophthalmology, Queen's Medical Centre, Nottingham, UK; ' Consultant dermatologist and dermatological surgeon, Department of Dermatology, Nottingham NHS Treatment Centre, Nottingham, UK

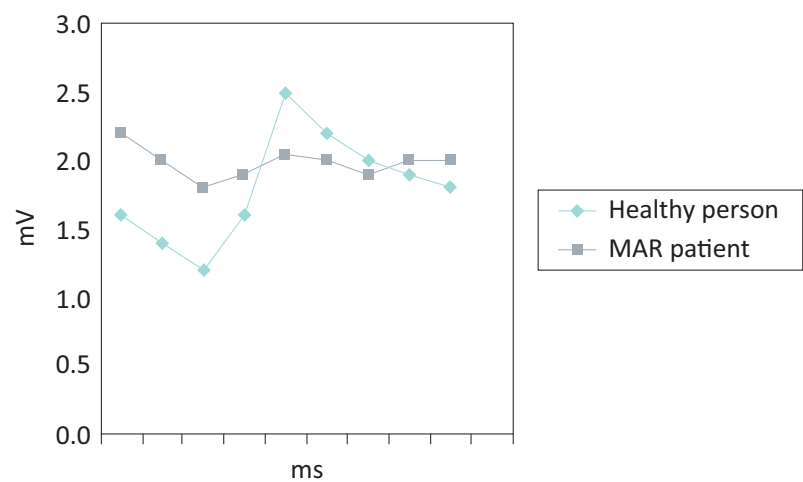

Fig 1. ERG shows significantly reduced amplitudes of rod response in MAR patient (grey line) in contrast to the amplitudes of a healthy person (green line). $E R G$ = electroretinography; $M A R=$ melanoma-associated retinopathy.

symptoms and past history, MAR was suspected and electrodiagnostics were arranged, demonstrating flat B waves. Flash electroretinography (ERG) indicated an abnormality in rod-mediated function at the inner retinal level (Fig 1). Pattern ERG suggested poor macular function, while visual field assessment suggested a development of anterior retinal antigens possibly related to his melanoma. A serum sample was sent to a laboratory in the US to confirm antibody detection.

A full body computerised tomography (CT) scan and magnetic resonance imaging (MRI) of the brain and orbits were arranged in light of the ophthalmic symptoms. The MRI was normal ruling out primary uveal malignant melanoma and ocular metastases (Fig 2a) while CT only identified a $2-\mathrm{cm}^{2}$ lymph node in the left axillary vault (Fig 2b). At follow-up the lymph node was palpable and was biopsied via fine needle aspiration under ultrasound guidance. Cytology revealed metastatic melanoma stage IIIb, thus a left axillary lymph node dissection followed.

The patient's serum test showed positivity for anti-retinal antibodies, believed to be linked to his metastatic melanoma. Visual disturbance had occurred due to melanoma recurrence in the left axilla, acting as a warning sign. After lymph node dissection, the patient's vision started to improve and has remained static and well tolerated. A diagnosis of melanomaassociated paraneoplastic retinopathy (MAR) was made. Follow-up ERG demonstrated an improvement in visual acuity. 


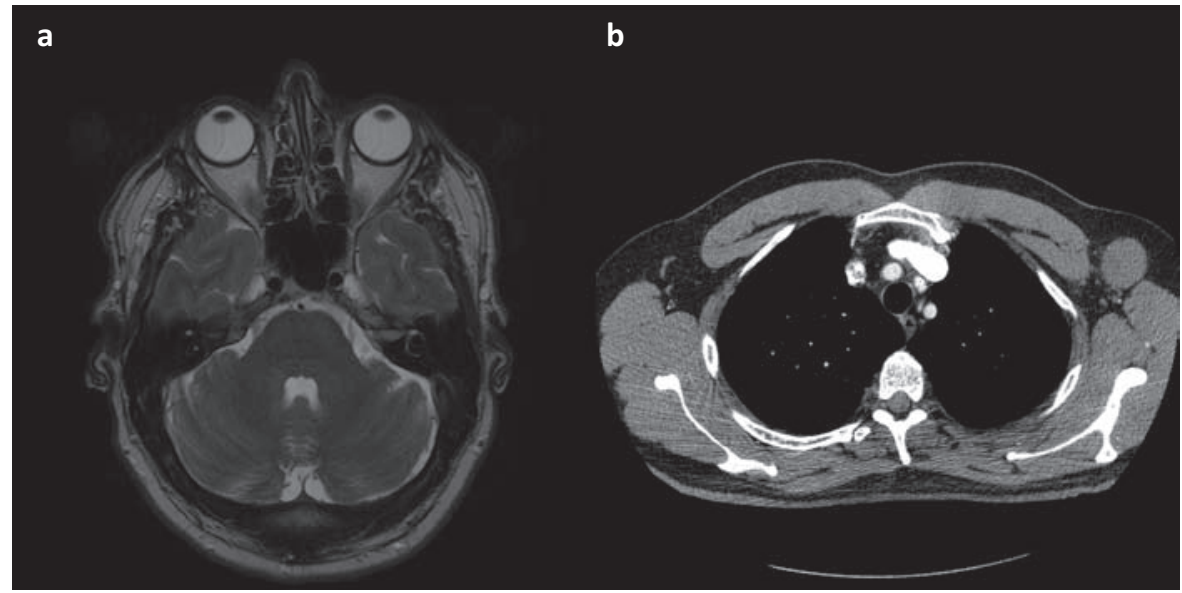

Fig 2. (a) Normal MRI brain; (b) CT thorax showing a $2 \mathrm{~cm}^{2}$ left axillary lymph node. $\mathrm{CT}=$ computerised tomography; $\mathrm{MRI}=$ magnetic resonance imaging.

\section{Discussion}

MAR is a rare paraneoplastic syndrome involving antibody production against tumour components (melanoma-associated antigens) which cross-react with the retinal bipolar cells. ${ }^{1,2}$ It was first reported and suggested to be a paraneoplastic phenomenon in a patient with a pre-existing diagnosis of melanoma who reported night blindness. ${ }^{2}$

The proposed mechanism involves a B-lymphocyte response against yet to be identified melanoma antigens that incite the production of autoantibodies that cross-react with specific retinal components. ${ }^{2,3}$ The serum of our patient showed high titres of specific antibodies against retinal cells. High titres of antibodies to retinal and optical nerve structures have been described in a similarly presenting case. ${ }^{4}$

Patients develop a sudden onset of shimmering, flickering or pulsating photopsias that begin on average 3.6 years (ranging 2 months to 19 years) after tumour diagnosis, and often complain of night blindness and a progressive loss of vision. ${ }^{4}$ Early diagnosis can be difficult due to subtle clinical and ophthalmoscopic findings. ERG is extremely sensitive in detecting abnormalities associated with MAR. Treatment options reported to improve visual symptoms include systemic steroids, plasmapharesis, interleukin-2 and dacarbazine chemotherapy, however the long-term effects remain undetermined. ${ }^{5}$

Coincidently, our patient was found to also suffer from non-segmental vitiligo affecting his hands, underlying his autoimmune predisposition. It is thought that vitiligo is an independent favourable prognostic factor in stage III and IV metastatic melanoma patients. ${ }^{6}$

Presence of MAR allowed early detection for metastatic disease in the left axilla. A multidisciplinary approach to this patient's care helped achieve a favourable outcome.

To allow timely diagnosis and the treatment of future patients, visual disturbance with a history of malignant melanoma should highlight the possibility of MAR in addition to brain metastasis.

\section{Learning points}

> MAR is a rare paraneoplastic complication of cutaneous malignant melanoma, typically presenting months to years after the initial diagnosis of melanoma but heralding the onset of metastatic disease

> Symptoms include persistent shimmering, pulsating or flickering photopsias of gradual onset, with or without night blindness

$>$ The natural history is said to be progressive visual loss but there are reports of prolonged stabilisation of symptoms following treatment with corticosteroids, plasma exchange or intravenous immunoglobulin

\section{References}

1 Ladewig G, Reinhold U, Thirkill CE et al. Incidence of antiretinal antibodies in melanoma: screening of 77 serum samples from 51 patients with American Joint Committee on Cancer stage I-IV. Br J Dermatol 2005;152:931-8.

2 Rahimy E, Sarraf D. Paraneoplastic optic and non-paraneoplastic retinopathy and neuropathy: evaluation and management. Surv Ophthalmol 2013;58:430-58.

3 Pfohler C, Haus A, Palmowski A et al. Melanoma-associated retinopathy: high frequency of subclinical findings in patients with melanoma. Br J Dermatol 2003;149:74-8.

4 Rush JA. Paraneoplastic retinopathy in malignant melanoma. Am J Ophthalmol 1993:115:J90-1.

5 Liu C-H, Wang N-K, Sun M-H. Melanoma associated retinopathy. Taiwan J Opthalmol 2014;4:184-8.

6 Quaglino, P, Marenco F, Osella-Abate S et al. Vitiligo is an independent favourable prognostic factor in stage III and IV metastatic melanoma patients: results from a single-institution hospital-based observational cohort study. Ann Oncol 2010;21:409-14.

Address for corresponding author: Dr A Aslam, Department of Dermatology, Nottingham NHS Treatment Centre, Lister Road, Nottingham NG7 2FT, UK

Email:a.aslam@doctors.org.uk 